\title{
Retrograde amnesia for previously acquired Pavlovian conditioning: UCS exposure as a reactivation treatment
}

\author{
RICK RICHARDSON, DAVID C. RICCIO, and HENRY MOWREY \\ Kent State University, Kent, Ohio
}

\begin{abstract}
Relatively little information is available on the stimulus conditions that can make old memory vulnerable to amnesic treatment. Three experiments employing a Pavlovian fear conditioning paradigm examined the effectiveness of a UCS exposure in reactivating a previously established memory. Reactivation of memory was indicated by increased susceptibility to retrograde amnesia (RA). Experiment 1A established that a noncontingent footshock (NCFS) $24 \mathrm{~h}$ after aversive conditioning, but immediately prior to hypothermia, was effective in reactivating the earlier established memory. This finding was further examined in Experiment 1B, in which it was demonstrated that severity of RA in groups reexposed to the conditioned fear cues, either before or after NCFS, was equivalent to that in subjects given NCFS only prior to hypothermia. Surprisingly, presenting footshock during reexposure to the fear cues (i.e., a brief retraining trial) also permitted induction of RA for old memory. While amnesia was comparable in subjects exposed to hypothermia following one of the four reactivation conditions (NCFS, NCFS/CUE, CUE/NCFS, CUE-FS), differences in memory strength were found among subjects not exposed to hypothermia after their reactivation treatment. Experiment 2 demonstrated that the temporal gradient of amnesia that is characteristic of new learning was also obtained for NCFS-reactivated memory. These findings provide evidence that previously acquired information can be activated by the aversive component of the original training episode, and that this active memory is vulnerable to an amnestic treatment.
\end{abstract}

In recent years there has been increasing evidence that retrograde amnesia (RA) for an old, wellestablished memory can be induced if the memory is reactivated (e.g., Misanin, Miller, \& Lewis, 1968; Schneider \& Sherman, 1968). Demonstrations that amnesia can be produced for presumably wellconsolidated information are important because they suggest that the vulnerability to RA may be more closely related to the degree of activity, rather than the age, of memory (Lewis, 1979). By and large, however, investigations have focused either on demonstrating the phenomenon or on comparing the characteristics of memory failure in new and old memory (Mactutus, Ferek, George, \& Riccio, 1982). Less attention has been paid to the stimulus conditions necessary to produce this type of amnesia. Among the few studies in this connection, Judge and Quartermain (1982), using anisomycin as an amnestic agent, reported a reactivation effect when, $3 \mathrm{~h}$ after training, mice were exposed to a chamber similar in size to the training apparatus, but RA for old memory was not obtained when a very different environment was used for cuing. A somewhat different ap-

This research was supported in part by NIMH Grant MH30223/ MH37535 to D.C.R. Reprints may be requested from Rick Richardson or David Riccio, Department of Psychology, Kent State University, Kent, Ohio $\mathbf{4 4 2 4 2 .}$ proach was taken by Gerson and Hendersen (1978) in one of the few studies to examine systematically the contribution of the components of the training episode. These investigators found that, for a punishment (passive avoidance) task, presentation of situational cues alone or in conjunction with footshock (FS) $24 \mathrm{~h}$ after training rendered memory susceptible to ECS. Neither FS in a different apparatus nor the different apparatus alone were sufficient. With an active avoidance task, the contextual apparatus cues were not sufficient unless either FS or the warning signal (CS) was presented. On the other hand, administering the FS or CS outside the training apparatus was ineffective.

The general aim of the present study was to explore further some of the stimulus conditions which could reactivate an earlier established memory, as indexed by an increased susceptibility to RA. If a posttraining treatment allows amnesia to be induced, it is a reasonable inference that reactivation was achieved and that at least some of the cues for retrieval of the target memory were present.

We chose to use Pavlovian rather than instrumental conditioning because the classical paradigm affords greater control over stimulus events during training and should therefore permit more direct manipulation of sources of reactivation. Because our Pavlovian paradigm involved differential fear conditioning to 
the brightness cues of a black-white chamber, the amount of shock and duration of the training session were necessarily greater than in the typical one-trial punishment task. Retrograde amnesia has been shown to diminish with increased training, so it seemed advisable to determine if amnesia could be obtained under these conditions. Accordingly, a pilot experiment was conducted to investigate the effects of a reliable amnestic agent, hypothermia, administered shortly after acquisition. Rats received deep body cooling (or not) following differential fear conditioning. The cooled animals showed significantly less passive avoidance of fear cues when tested $24 \mathrm{~h}$ later than did retention controls. This finding indicates that despite the use of more extended training, the Pavlovian conditioned response was susceptible to RA.

\section{EXPERIMENT 1A}

A growing body of research indicates that administration of noncontingent footshock (NCFS) to aversively trained subjects will reactivate memory. For example, NCFS has been effective in alleviating forgetting in young animals (Riccio \& Haroutunian, 1979; Spear \& Parsons, 1976) as well as in reversing performance decrements from amnestic treatments and other sources (Klein \& Spear, 1970; Miller \& Springer, 1972; Rescorla \& Heth, 1975). In these cases, however, the function of NCFS was to eliminate or reduce an already existent memory deficit. This class of findings is potentially quite different from that found from an approach in which an established (and presumably intact) memory is reactivated by NCFS exposure in order to increase its vulnerability to traumatic insult. Relatively few reports are directed at the latter problem. Gerson and Hendersen's (1978) data suggest that NCFS is not effective, as ECS failed to induce RA unless the FS was presented in conjunction with the training cues. On the other hand, a second FS has been shown to extend the temporal gradient of ECS, suggesting RA for earlier acquisition (Schneider \& Sherman, 1968). Furthermore, although they employed a somewhat atypical paradigm, Meyer and his colleagues (Howard, Glendenning, \& Meyer, 1974; Robbins \& Meyer, 1970) demonstrated that reexposure to a FS-escape contingency shortly prior to ECS results in suppression of memory for an earlier acquired discriminative shock-escape habit. Thus, these mixed findings leave ambiguous the status of the vulnerability of old memory following NCFS. However, there seems little doubt that the original UCS (footshock) should be a good candidate for reactivating memory of an aversive episode, whether one emphasizes the role of arousal (Schneider \& Sherman, 1968), motivational states (Meyer, 1972), or attributes of the target memory (Spear, 1973, 1978). The purpose of Experi- ment 1A was to determine whether RA for 24-h-old Pavlovian conditioning could be achieved by exposing subjects to a noncontingent footshock immediately prior to deep body cooling.

\section{Method}

Subjects. Twenty 123-130-day-old adult male naive rats, purchased from the Holtzman Company, served as subjects. The animals were individually housed in standard wire-mesh cages and ear-punched for identification purposes. Food and water were available ad lib. The subjects were maintained on a 15-/9-h (onoff) light-dark cycle. Training and testing occurred during the light part of the cycle between 12:00 and 2:30 p.m.

Apparatus. The training and testing apparatus was a $38 \times 18 \times$ $21 \mathrm{~cm}$ Plexiglas box divided into two equal-sized chambers. One chamber and its lid were painted black. The other chamber was painted white but had a clear Plexiglas lid. A 15-W light bulb was suspended $30 \mathrm{~cm}$ above the center of the white compartment. A guillotine doorway $(8 \times 8 \mathrm{~cm})$ connected the two compartments. The floor of the apparatus consisted of $2.5-\mathrm{mm}$ stainless steel grids spaced $1 \mathrm{~cm}$ apart. The grids of the black compartment were connected to a matched impedance shock source (Campbell \& Teghtsoonian, 1958) through a BRS/LVE (Model SC-922) scrambler. A white-noise generator was used to help mask extraneous sounds.

The NCFS apparatus consisted of a $18.5 \times 15.5 \times 20 \mathrm{~cm}$ unpainted wooden box. The floor consisted of 2-mm stainless steel grids spaced $1 \mathrm{~cm}$ apart. The grids were connected to the same type of matched impedance shock source used for conditioning; however, a slightly different scrambler (BRS/Foringer Model SC901) was employed. The NCFS box was located in a different room from that which housed the training and testing apparatus; illumination was provided by overhead fluorescent lights, and no white noise was present.

Procedures. For 3 days prior to the start of the experiment, each animal was handled 2-3 min daily. The fear-conditioning procedure consisted of four consecutive 2-min exposures to the discriminative color cues (i.e., B, W, B, W). During each period in the black compartment, six inescapable footshocks $(150 \mathrm{~V}, 1 \mathrm{sec})$ were delivered on a variable time schedule (after 10, 30, 40, 70, $90,100 \mathrm{sec}$ ). No shocks were administered during the two periods in which the animal was confined in the white compartment. Thus, a training session was $8 \mathrm{~min}$ long, and a total of 12 shocks were administered. The animals were randomly divided into two treatment groups. All subjects received Pavlovian differential fear conditioning on Day 1 followed $24 \mathrm{~h}$ later by NCFS. The NCFS consisted of a 3-sec, 150-V scrambled shock. One group of subjects (Train/NCFS/Hypo) received hypothermia immediately after the NCFS, whereas the other group (Train/NCFS) received no further treatment. Hypothermia treatment consisted of immersion (to the neck) in $3^{\circ}-4^{\circ} \mathrm{C}$ water until body temperature, as measured by a Yellow Springs telethermometer and rectal probe inserted $3.2 \mathrm{~cm}$, dropped to $21^{\circ} \mathrm{C}\left( \pm 1^{\circ} \mathrm{C}\right)$.

All subjects were tested for fear of the black compartment $24 \mathrm{~h}$ after the NCFS had been administered. Testing consisted of placing the subjects on the white side of the apparatus facing away from the door, which was closed. After $10 \mathrm{sec}$, the door was raised and the latency taken to enter (all four paws) the black compartment for the first time was recorded. A second measure, the total time spent on the white (safe) side (TTW) during the entire 10-min test, was also obtained. Long latency and high TTW scores are indicative of strong retention of fear. The experimenter was not aware of what treatment conditions the subjects had received.

\section{Results and Discussion}

The median cross-through latencies were $325 \mathrm{sec}$ (range 206-539) for the reactivation-only condition 
and $99 \mathrm{sec}$ (range 31-186) for the group receiving reactivation followed by hypothermia. The total time on the safe side measure showed a similar effect: median TTW scores were 549 (range 380-592) and $296 \mathrm{sec}$ (range 239-420) for the Train/NCFS and Train/NCFS/ Hypo groups, respectively.

Separate Mann-Whitney $U$ tests on the latency and TTW data indicated that the subjects receiving hypothermia after the NCFS had reliably less fear of the black compartment than did subjects that had not received hypothermia after the NCFS (Train/ NCFS/Hypo vs. Train/NCFS; $U=0$ for latency measure and $U=2$ for TTW measure, both ps $<.002$ ).

These data extend previous work by demonstrating that RA can be induced for an old memory based upon Pavlovian differential conditioning. Of particular interest is the fact that the reactivation treatment was exposure to footshock rather than to the conditioned fear cues. Thus, memory became susceptible to an amnestic treatment following noncontingent administration of the UCS component of training. This finding is unusual in that NCFS has often been used successfully to promote recovery from forgetting (Miller \& Springer, 1972; Riccio \& Haroutunian, 1979; Spear \& Parsons, 1976), but relatively seldom as a means for reactivating an intact memory in order to induce RA. The parallelism between these phenotypically "opposite" outcomes is not without interest, of course.

\section{EXPERIMENT 1B}

Several studies have found that prior exposure to one or more of the components of training will attenuate the effectiveness of RA treatments (Hinderliter \& Riccio, 1977; Hinderliter, Smith, \& Misanin, 1973; Jensen \& Riccio, 1970; Nachman \& Meinecke, 1969). A particularly salient example of this type of familiarization is seen when amnestic subjects are reexposed to the training/amnestic event. For example, Riccio and Stikes (1969) obtained no detectable retention loss in rats following the second sequence of onetrial punishment training/hypothermia, and similar outcomes have been reported for other agents (Kesner, McDonough, \& Doty, 1970; Nachman \& Meinecke, 1969). Thus, the evidence that FS in this paradigm reactivated memory sufficiently to render it vulnerable to amnesia is of interest, since the FS might have functioned akin to further training and strengthened memory rather than permitting RA. The aim of Experiment $1 B$ was to determine directly whether RA for old memory could be induced when the reminder FS was administered in the presence of the original $\mathrm{CS}+$, (i.e., an abbreviated retraining trial) and to compare this with the RA induced when the reminder FS was given noncontingently. An additional aim was to determine whether the reactivation induced by
NCFS was modulated by an extinction exposure to the CS+. Because order of presentation of events could be important, half of the subjects in this condition received cue exposure followed by NCFS and half received the same treatments in reverse order.

\section{Method}

Subjects and Apparatus. Sixty-three naive adult male rats, ranging in age from 97 to 158 days (purchased from the Holtzman Company), served as subjects. The animals were housed and treated in an identical fashion as those in the previous experiment. The same apparatus employed in Experiment $1 \mathrm{~A}$ was used.

Procedure. Handling, conditioning, cooling, and testing procedures were the same as those described for Experiment 1A. The NCFS reactivation treatment was the same as that used in Experiment 1A. The cue reexposure reactivation treatment was a 20-sec nonreinforced exposure to the black compartment.

On Day 1, all animals received Pavlovian differential fear conditioning. The animals were then divided randomly into seven treatment conditions. All subjects received one of four different reactivation treatments, and some subjects were given hypothermia immediately after reactivation. All reactivation treatments, and hypothermia if it was administered, occurred on Day 2. One group of subjects was given an NCFS and then reexposed to the fear cues. Half of these animals were given hypothermia (NCFS/Cue/ Hypo) immediately after the cue exposure; the remainder were not (NCFS/Cue). Another group was reexposed to the cues prior to receiving NCFS. Again, half of these subjects received hypothermia (Cue/NCFS/Hypo) immediately after the NCFS, whereas the remainder did not (Cue/NCFS). (One rat in the former condition died and was not replaced.) The NCFS and cue exposure were separated by 5-10 sec in these four conditions. Two additional groups of subjects were given a 1-sec footshock at the end of the 20-sec cue exposure. This footshock occurred in the training apparatus. Thus, this "reactivation" treatment can be construed as a retraining trial. Half of these subjects (Cue-FS/Hypo) received hypothermia immediately after the termination of the footshock, whereas the remainder did not (Cue-FS). Finally, one group of subjects (NCFS/Hypo) was not reexposed to the fear cues but was given a single NCFS followed immediately by hypothermia. This group constituted a replication of the experimental condition in Experiment 1A. Testing was performed on Day 3, and the experimenter was unaware of what treatment condition any subject was in.

\section{Results and Discussion}

The results are presented in Figure 1. Separate Kruskal-Wallis ANOVAs on the latency and TTW data indicated a significant treatment effect $(\mathrm{H}>45.4$ for both measures, $\mathrm{p}<.001$ in each case). Pairwise comparisons were made of groups that received the same reactivation treatment followed by hypothermia or not. With either the latency or TTW measure, it was consistently found that animals that received hypothermia after a particular reactivation treatment demonstrated poorer retention than did subjects that did not receive hypothermia after the same reactivation treatment (all Us $\leqslant 4$, all ps $\leqslant .002$ ). For instance, the Cue-FS/Hypo group had less fear of the black compartment than did the Cue-FS group ( $U=0$ for both latency and TTW measures, ps $<.002$ ). Furthermore, the NCFS/Hypo group differed from all three groups not receiving hypothermia after a 

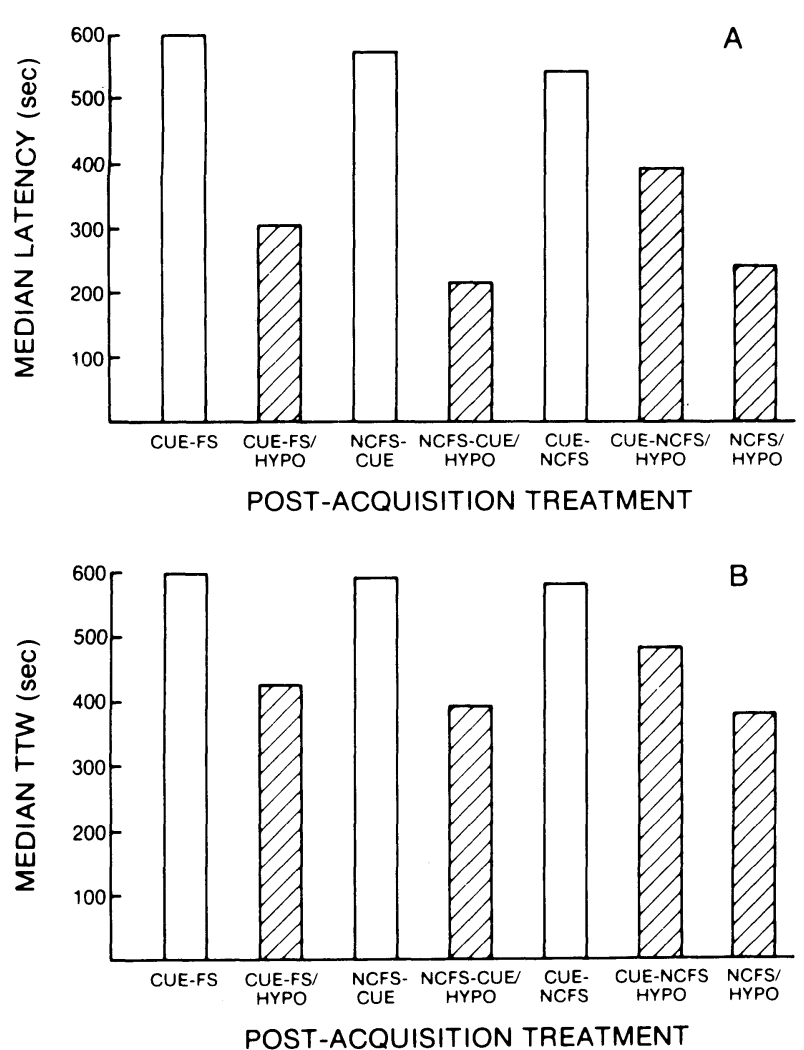

Figure 1. Retention of previously conditioned fear when various reactivation manipulations are followed (or not) by amnestic treatment (hypothermia). The designation FS refers to footshock given in the training apparatus and NCFS to noncontingent footshock administered in a different apparatus. See text for details. Latencies are presented in Panel A and time on safe side in Panel B.

reactivation treatment (all Us $=0$ for both latency and TTW measures, ps $<.002$ ). As the Us indicate, the distribution of scores was nonoverlapping for several of the comparisons of reactivation groups with and without hypothermia.

Additional Kruskal-Wallis ANOVAs comparing the groups that did or did not receive hypothermia after their respective reactivation treatment indicated significant differences among the groups receiving reactivation only $(\mathrm{H}=6.08$ for latency measure and $\mathbf{H}=7.29$ for TTW, both ps $<.05$ ), but no differences among groups that received hypothermia after reactivation $(\mathrm{H}=3.60$ for latency and $\mathrm{H}=2.98$ for $\mathrm{TTW}$, both $\mathrm{ps}>.10$ ). These analyses suggested that hypothermia was effective in producing an equivalent amount of RA irrespective of the reactivation treatment. Furthermore, these analyses suggested that the reactivation treatments affected memory differentially. The differences, while small, were reliable. Pairwise comparisons indicated that the Cue-FS treatment resulted in better test performance than the other two treatments: With the latency measure, the Cue-FS group differed from the NCFS/Cue group $(U=14, p=.02)$, and with the TTW measure the
Cue-FS differed from both the NCFS/Cue $(U=13$, $\mathrm{p}<.02)$ and the Cue/NCFS groups $(U=15.5, \mathrm{p}<$ .05). Receiving the footshock in the original training context resulted in better retention of the conditioned fear than receiving a footshock in a different context either before or after being exposed to the conditioning cues. Apparently, the brief exposure to the CS complex alone had some extinction effect.

It is clear from these data that all four reactivation manipulations were effective in making a previously learned response susceptible to amnesia. The data replicate Experiment $1 \mathrm{~A}$ by demonstrating that administration of footshock outside the training apparatus is sufficient to reactivate earlier learned fear, as reflected in the memory loss induced by deep body cooling. Furthermore, the addition of a brief nonreinforced exposure to the CS+ either before or after the NCFS had a slight effect on retention in controls but no apparent influence on the severity of RA. Perhaps more surprisingly, when earlier learning was reactivated by a direct re-pairing of the CS+ and shock, memory was as vulnerable to amnesia as in the other conditions. This group was originally included in anticipation of the possibility that an increase in strength of learning produced by reactivation might tend to reduce the severity of RA. The re-pairing condition, by receiving an explicit, but abridged, version of training, should provide an estimate of the extent to which RA for old memory is counteracted by further learning. But our expectation of attenuated RA was not confirmed, as hypothermia-induced RA following a CS-UCS presentation was comparable to that in the NCFS group. Although this result appears to contrast with other research showing a sharp increment in memory when the training-amnestic treatment is repeated (Kesner, et al., 1970; Nachman \& Meinecke, 1969; Riccio \& Stikes, 1970), two important differences between the paradigms should be noted. First, the present study employed an abridged portion of the original training session, rather than an identical trial. Second, subjects in this experiment were exposed to the amnestic agent for the first time after the reactivation treatment, whereas subjects in the earlier studies had already been exposed to the amnestic agent on at least one previous occasion. In this connection it should be noted that there are data suggesting that reactivated memory may be unusually "sensitive," that is, vulnerable to amnestic treatments that are ineffective for new learning (Mactutus, Riccio, \& Ferek, 1979; Mactutus et al., 1982). Any of these factors may be responsible for the difference.

\section{EXPERIMENT 2}

In the preceding experiments, each group of subjects receiving hypothermia exhibited an impairment in retention of a Pavlovian conditioned response. It has been demonstrated previously that memory must be cued or reactivated in order to obtain RA (cf. 
Gerson \& Hendersen, 1978; Mactutus et al., 1982), so a control group receiving cooling without reactivation was not included. However, the surprising effectiveness of the hypothermia treatment in all reactivation conditions raised the concern that the amnestic agent may have been influencing some aspect of the test performance rather than memory.

When only the CS is used as the reactivation treatment, the strength of amnesia shows a temporal gradient, diminishing with increased delay between reactivation and hypothermia (Mactutus et al., 1982). This outcome effectively counters a performance artifact explanation of the retention deficit. But since the present reactivation treatments included the UCS, it is possible that the combined effects of the shock and cooling somehow altered performance at testing. While such an outcome seemed unlikely, demonstration of a temporal gradient of amnesia for shockreactivated old memory would provide empirical evidence for a memorial interpretation. Thus, in Experiment 2 , subjects received a NCFS as a reactivation treatment followed by hypothermia after delay intervals of $.25,30$, or $120 \mathrm{~min}$. If the retention loss seen in Experiments $1 \mathrm{~A}$ and $1 \mathrm{~B}$ was indeed an amnesic effect, then the vulnerability of reactivated memory should diminish as a function of the delay of treatment.

\section{Method}

Subjects. Thirty-six 63-114-day-old adult male naive rats, purchased from the Holtzman Company, served as subjects. The animals were housed and maintained in an identical fashion as those in the previous experiments. The apparatus employed was the same as that used in Experiment 1A.

Procedures. Handling, conditioning, cooling, and testing procedures were the same as those described for Experiment 1A. One day after the Pavlovian training, all subjects received a single NCFS (same as that used in Experiment 1A) as a reminder treatment. All subjects also received hypothermia after the reactivation treatment. The three groups of subjects differed in the interval separating the NCFS and hypothermia. Some subjects received hypothermia $.25 \mathrm{~min}$ after reactivation, others after $30 \mathrm{~min}$ had elapsed, and a final group $120 \mathrm{~min}$ after reactivation. (All Ns were 12, with the exception of the 30-min-delay group, in which one rat was discarded due to experimenter error.)

\section{Results and Discussion}

The latency data, depicted in Figure 2, suggest that test performance improves as the delay between NCFS and hypothermia increases. A Kruskal-Wallis ANOVA verified a significant treatment effect $(H=6.37$, $\mathrm{p}<.05)$. Subjects receiving hypothermia immediately after reactivation showed poorer retention than those receiving hypothermia after a 120 -min delay $(U=32.5$, $\mathrm{p}<.05$ ). The 30 -min-delay group was intermediate and failed to differ from the other two conditions. A quite similar, but shallower, gradient, which did not reach conventional levels of significance, was obtained with the TTW measure $(\mathrm{H}=5.25, \mathrm{p}>.05$ but $<.10)$.

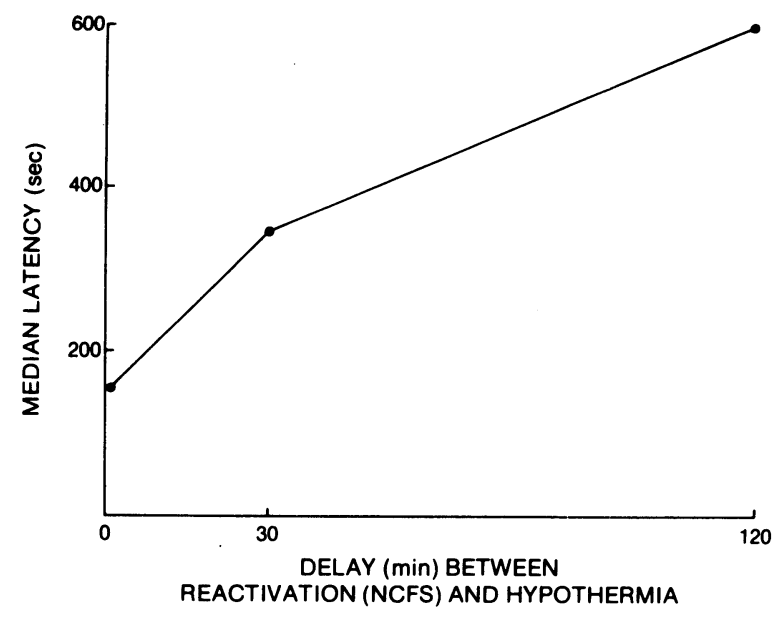

Figure 2. Retention of fear as a function of the interval between reactivation treatment (NCFS) and hypothermis.

These data indicate that a temporal gradient of susceptibility to RA can be obtained for old memory when the learning was based upon Pavlovian conditioning rather than instrumental punishment (cf. Randall \& Riccio, 1969) and when the UCS serves as the reactivation treatment. Thus, these findings replicate and extend the data based upon NCFS in Experiment 1.

Experiment 2 provides further support for the notion that the extent to which memory is active, rather than its age per se, determines its susceptibility to an amnestic treatment. Reactivation by NCFS permits RA to be induced, but only for a limited time span. An old reactivated memory apparently "subsides," in the sense of becoming less modifiable by the amnestic agent, much as does new memory (Mactutus et al., 1982). At the same time, these findings of temporal dependency demonstrate that the retention deficits obtained in this study are not attributable to either nonspecific retroactive interference from exposure to deep body cooling or a performance artifact at the time of testing.

\section{GENERAL DISCUSSION}

The present findings provide further support for the view that memory, whether new or old, is vulnerable to amnesia when in an active state (cf. Lewis, 1979; Mactutus et al., 1982). RA was induced by administering hypothermia immediately after a reactivation treatment (NCFS), but not when cooling was separated from the reactivation by a 2-h delay. This temporal gradient rules out any proactive effect of hypothermia on performance at testing. Moreover, since it is generally believed that retroactive interference is relatively independent of the interval between acquisition of the target information and the interfer- 
ing episode (Newton \& Wickens, 1956), the temporal dependency makes unlikely an interpretation based upon nonspecific interference effects (cf. Wickens, Tuber, Nield, \& Wickens, 1977).

Of particular interest is the fact that previously acquired information was made active by presentation of the UCS employed in the original fear conditioning task. This manipulation has the practical advantage of not introducing extinction into the reactivation treatment. Moreover, the potency of the UCS as a reactivation treatment is indicated by the finding that severity of RA was not altered by inclusion of the CS. This relationship is strikingly similar to the pattern reported in a quite different paradigm (Riccio \& Haroutunian, 1979). Using a reinstatement manipulation to alleviate developmental forgetting, these investigators found that a single NCFS midway through the retention interval improved retention as much as either NCFS followed by a CS exposure or an actual re-pairing of CS and UCS. Despite the obvious differences between paradigms, and the fact that one involved inducing amnesia while the other reduced forgetting, a reasonable inference is that both involved the reactivation of earlier Pavlovian learning. Thus, it appears that a substantial proportion of the attributes of fear conditioning can be evoked by the original reinforcing stimulus. A threshold notion could then explain why the presentation of additional cues (CS+, environmental context) is not necessary. An intriguing, but more speculative, possibility is that a salient reminder stimulus can "overshadow" other stimuli; such a phenomenon has been found to occur, of course, when compound CSs are used in Pavlovian conditioning (MacKintosh, 1974).

The evidence that the RA gradient can be extended by a second FS is consistent with Schneider and Sherman's (1968) data. However, it should be noted that their "noncontingent" footshock treatment was probably closer to the re-pairing condition in Experiment 1B. In the Schneider and Sherman study, FS was delivered independently of the previously punished response, but in the presence of the original training context (apparatus cues). But inducing vulnerability to RA by an NCFS does differ from the outcome obtained by Gerson and Hendersen (1978). These investigators failed to find RA for prior passive or active avoidance learning when FS was given outside the training situation. One plausible explanation for this difference is that our use of multiple shocks with a static (brightness) cue may have permitted the UCS to function also as a CS. That is, although the black compartment was the best predictor of aversive stimuli, the initial shock and the concomitant internal changes may become a signal for further shocks (cf. Concannon, Riccio, \& McKelvey, 1980).

\section{REFERENCES}

Campeell, B. A., \& Teghtsoonian, R. Electrical and behavioral effects of different types of shock stimuli on the rat. Journal of Comparative and Physiological Psychology, 1958, 51, 185-192.

Concannon, J. T., Riccio, D. C., \& McKelvey, J. Pavlovian conditioning of fear based upon hormonal mediation of prior aversive experiences. Animal Learning \& Behavior, 1980, 8, 75-80.

Gerson, R., \& Hendersen, R. W. Conditions that potentiate the effects of electroconvulsive shock administered 24 hours after avoidance training. Animal Learning \& Behavior, 1978, 6, 346-351.

Hinderliter, C. F., \& Riccio, D. C. Long-term effects of prior experience in attenuating amnesia. American Journal of Psychology, 1977, 90, 407-418.

Hinderliter, C. F., Smith, S. L., \& Misanin, J. R. Effects of pretraining experiences on retention of a passive avoidance task following ECS. Physiology \& Behavior, 1973, 10, 671-675.

Howard, R. L., Glendenning, R. L., \& Meyer, D. R. Motivational control of retrograde amnesia: Further explorations and effects. Journal of Comparative and Physiological Psychology, 1974, 86, 187-192.

Jensen, R. A., \& Riccio, D. C. Effects of a prior experience upon retrograde amnesia induced by hypothermia. Physiology \& Behavior, 1970, 5, 1291-1294.

JudGe, M. E., \& Quartermain, D. Characteristics of retrograde amnesia following reactivation of memory in mice. Physiology \& Behavior, 1982, 28, 585-590.

Kesner, R. P., McDonough, J. H., \& Doty, R. W. Diminished amnesia effects of a second electroconvulsive seizure. Experimental Neurology, 1970, 27, 527-533.

KLe IN, S. B., \& SPEAR, N. E. Reactivation of avoidance learning memory in the rat after intermediate retention intervals. Journal of Comparative and Physiological Psychology, 1970, 72, $498-504$.

LEwIs, D. J. Psychobiology of active and inactive memory. Psychological Bulletin, 1979, 86, 1054-1083.

MACKInTosh, N. J. The psychology of animal learning. London: Academic Press, 1974.

Mactutus, C. F., Ferex, J. M., George, C. A., \& Riccio, D. C. Hypothermia-induced amnesia for newly acquired and old reactivated memories: Commonalities and distinctions. Physiological Psychology, 1982, 10, 79-95.

Mactutus, C. F., Riccio, D. C., \& Ferex, J. M. Retrograde amnesia for old (reactivated) memory: Some anomalous characteristics. Science, 1979, 204, 1319-1320.

Meyer, D. R. Access to engrams. American Psychologist, 1972, 27, 124-133.

Mille R, R. R., \& Springer, A. D. Induced recovery of memory in rats following electroconvulsive shock. Physiology \& Behavior, 1972, 8, 645-651.

Misanin, J. R., Miller, R. R., \& Lewis, D. J. Retrograde amnesia produced by electroconvulsive shock after reactivation of a consolidated memory trace. Science, 1968, 160, 554-555.

Nachman, M., \& Meinecke, R. O. Lack of retrograde amnesia effects of repeated electroconvulsive shock and carbon dioxide treatments. Journal of Comparative and Physiological Psychology, 1969, 68, 631-636.

Newton, J. M., \& Wickens, D. D. Retrograde inhibition as a function of the temporal position of interpolated learning. Journal of Experimental Psychology, 1956, 51, 149-154.

Randall, P. K., \& Riccio, D. C. Fear and punishment as determinants of passive-avoidance responding. Journal of Comparative and Physiological Psychology, 1969, 69, 550-553.

Rescorla, R. A., \& Heth, D. C. Reinstatement of fear to an extinguished conditioned stimulus. Journal of Experimental Psychology: Animal Behavior Processes, 1975, 1, 88-96.

Riccio, D. C., \& Haroutunian, V. Some approaches to the 
alleviation of ontogenetic memory deficits. In N. E. Spear \& B. A. Campbell (Eds.), Ontogeny of learning and memory. Hillsdale, N.J: Erlbaum, 1979.

Riccio, D. C., \& Stikes, E. R. Persistent but modifiable retrograde amnesia produced by hypothermia. Physiology \& Behavior, $1969,4,649-652$.

Robbins, M. J., \& MEYer, D. R. Motivational control of retrograde amnesia. Journal of Experimental Psychology, 1970, 84, 220-225.

Schneider, A. M., \& Sherman, W. Amnesia: A function of the temporal relation of footshock to electroconvulsive shock. Science, 1968, 159, 219-221.

Spear, N. E. Retrieval of memory in animals. Psychological Review, 1973, 80, 163-194.
SPEAR, N. E. The processing of memories: Forgetting and retention. Hillsdale, N.J: Erlbaum, 1978.

Spear, N. E., \& Parsons, P. Analysis of a reactivation treatment: Ontogeny and alleviated forgetting. In D. Medin, R. Davis, \& W. Roberts (Eds.), Coding processes in animal memory. Hillsdale, N.J: Erlbaum, 1976.

Wickens, D. D., Tuber, D. S., Nield, A. F., \& Wickens, C. Memory for the conditioned response: The effects of potential interference introduced before and after original conditioning. Journal of Experimental Psychology: General, 1977, 106, 47-70.

(Manuscript received October 28, 1982; revision accepted for publication January $7,1983$. 